\title{
A Multisensory 3D Environment as Intervention to Aid Reading in Dyslexia: A Proposed Framework
}

\author{
Martyn Broadhead, Damon Daylamani-Zad, Lachlan Mackinnon, Liz Bacon \\ Department of Computing and Information Systems \\ University of Greenwich \\ London, United Kingdom \\ \{M.P.Broadhead, D.D.Zad, L.Mackinnon, E.Bacon\}@ greenwich.ac.uk
}

\begin{abstract}
Developmental Dyslexia (DD) is a common language-based learning difficulty which occurs across all cultures. Whilst various interventions are implemented to aid with reading difficulties, research suggests that phonics is still the most promising approach, yet the challenge in this approach has always been keeping pupils engaged and interested. Multisensory approaches have shown promise in keeping pupils engaged but they are time consuming and require high levels of teacher involvement. This paper suggests using 3D environments and gaming technology as a multisensory intervention to aid reading in Dyslexia. The paper proposes an initial framework and indicates the development and evaluation strategy for the framework.
\end{abstract}

Keywords-Dyslexia, VAKT, Multisensory Intervention, Gaming technology, 3D environment

\section{INTRODUCTION}

Developmental Dyslexia (DD) is a common languagebased learning difficulty which occurs across all cultures [1], estimates of prevalence vary from country to country as they are affected by factors such as school provision and orthography of the language. The British Dyslexia Association (BDA) estimates that $10 \%$ of the population are dyslexic, with $4 \%$ being severely affected [2]. Dyslexia identifies a difficulty with words, dys meaning difficult, and lexia related to words /reading, or language [3]. The difficulty is not easily attributed to poor schooling, intellectual deficits or sensory disorders [4][5]. An array of theories have been forwarded as to the cause of dyslexia, the current consensus is that it is neurodevelopmental in origin. Functional and structural studies of the brain have identified anomalies which are present in embryonic and foetal growth, with dysfunction in an area of the left hemisphere being focused in the ventral-dorsal-anterior, this an area normally associated with reading and phonological processing, [6]. In dyslexia atypical patterns of hemisphere activation have been identified [7].

There have been various interventions and research to aid with reading difficulties in education and psychology. Yet literature suggests that practitioners consider phonics to be the most promising approach. The issue with phonics is that students can lose interest and do not engage at the levels they would need in order to be successful. Multisensory approaches, specifically VAKT (Visual, Auditory, Kinesthetic and Tactile) have shown increased engagement and show promising results with students. However there are issues with cost and teacher involvement that affect the accessibility and availability of such methods.

This paper proposes use of computer assisted intervention in the form of a gamified 3D environment as an approach to implementing multisensory interventions to aid with reading. The paper presents a detailed discussion on the arguments in effective methods for learning to read (section II), then in section III presents a review of current reading interventions. Section IV reviews the use of $3 \mathrm{D}$ virtual environments as interventions and then presents an initial framework for implementing a gamified 3D environment for implementing a multisensory intervention (Section V). The development and testing plans for the proposed framework are discussed in section VI before the concluding discussions in section VII.

\section{LEARNING TO READ}

There is argument as to the most effective method of teaching children to read, this is illustrated by what has come to be known as the "reading wars"[8], [9]. At the core of the argument is a debate as to whether literacy is best taught through codification or comprehension of language.

Traditionalists suggest that reading is best achieved through decoding text using phonics, a systematic method requiring the learner to decode and understand the relationship between phonemes and graphemes [10], [11] . The progressive approach suggests reading is best achieved by a whole word or language based method, placing the emphasis on comprehension and understanding. Goodman [12], argues that reading is accomplished by a similar means to learning spoken language, the reader learns to read based on their needs, and the reader responds to "authentic literary events that meet their needs". This calls into question whether it is possible to learn to read through exposure to literature without systematic teaching of phoneme to grapheme relationships.

The US National Reading Panel Report 2000 [13] gathered research from a range of studies to evaluate 5 areas which the panel considered central to standard reading instruction. The panel identified these areas; Alphabetics (Phonemic Awareness \& Phonics Instruction), Fluency, Comprehension, Teacher Education and Reading Instruction, and Computer Technology and Reading Instruction.

Evaluation of 52 studies found that incorporating phonemic awareness into reading instruction was more effective than methods that omitted phonemics. The panel concluded that 
explicit instruction in phonemic awareness should be a central component of reading instruction. Phonics instruction is the most highly investigated intervention, and it is also the only approach whose success has been statistically confirmed in both children and adolescents [14]. These findings present a particular challenge to reading instruction for learners with dyslexia because there is evidence that dyslexic learners have a particular difficulty with decoding phonics [15]-[18].

Reading interventions are implemented when learners are not working at an age appropriate level, the interventions map closely to the areas investigated by the national reading panel. The precise nature of intervention would be determined by assessment individual student needs. Areas for intervention include [19]:

1) Phonemic awareness (Sound awareness)

2) Phonics (Links between sound and letters)

3) Spelling/writing (Correctly composing words)

4) Fluency (Ability to read accurately and fluently)

5) Vocabulary (Meaning and how to pronounce words)

6) Comprehension (Ability to understand)

The Rose Report 2009 [20] in the UK and the US National Reading Panel 2000 [13] both endorse multisensory interventions. A multisensory intervention would not by definition require the use of computer technologies. A multisensory approach uses a range of sensory modalities summed up as "hear it, see it, say it and write it" [21]. An early exploration of the approach was implemented by Fernald [22] who developed a multimodal method to assist children with learning difficulties to recognise words.

A multisensory approach may be identified using the acronym, VAKT, it may simultaneously utilise a range of four sensory modalities [23]-[26]; Visual (V), Auditory (A) Kinesthetic $(\mathrm{K})$ and Tactile $(\mathrm{T})$. The consensus of opinion on Dyslexia is that it is neurobiological in origin [27]. Standard reading and processing of language uses the left hemisphere. In dyslexia atypical patterns of hemisphere activation have been identified [7]. The TEA Dyslexia Handbook [28] advises that teaching must employ a multisensory approach to simultaneously to utilise broader range of neural pathways.

\section{3D VIRTUAL ENVIRONMENTS AS INTERVENTIONS}

The ability of information systems to store, collect, process, and represent data is formidable in facilitating and supporting a range of pedagogic approaches [29]. 3D virtual environments present a unique toolset, which blends, human computer interfaces (HCI), networking, 3-dimensional graphics and sensory technology. A 3D environment promotes an immersive experience, which can represent a realistic or space based 3D situation. The ability to monitor stimulus, and control the environment are features of 3D environments, which aid the quantification of results. 3D virtual environments have been utilised and shown to be effective in a range of areas; in special needs such as autism [30], managing learning difficulties [31], pain management [32], [33], cognitive impairments [34], [35] and in clinical applications [36], [37].

The development of interaction methods for 3D environments, such as voice inputs, motion sensing, spatial interaction and immersive virtual reality headsets is changing how games are played. If applied to education, there is potential for immersive multisensory interventions to support dyslexic students. 3D Virtual Environments can be used not just to enhance traditional methods of teaching literacy but may form a more transformational pedagogy [38], yet there is little empirical work in this area. This is compounded by the limited number of studies, which have looked specifically at dyslexia and 3D virtual environments. Reviews have found only seven significant studies, only three of these were interventions to aid literacy [39].

\section{A PROPOSED FRAMEWORK FOR 3D ENVIRONMENTS AS INTERVENTIONS}

This paper proposes a framework for a gamified 3D environment as an intervention for dyslexia, illustrated in Fig. 1. It utilises multisensory techniques with phonics and phonemic awareness based teaching methodologies. The objective of the game is to craft words/sentences from audible target words/sentences using collected resources. This will address the reading intervention areas of alphabetic, spelling, vocabulary, comprehension and fluency.

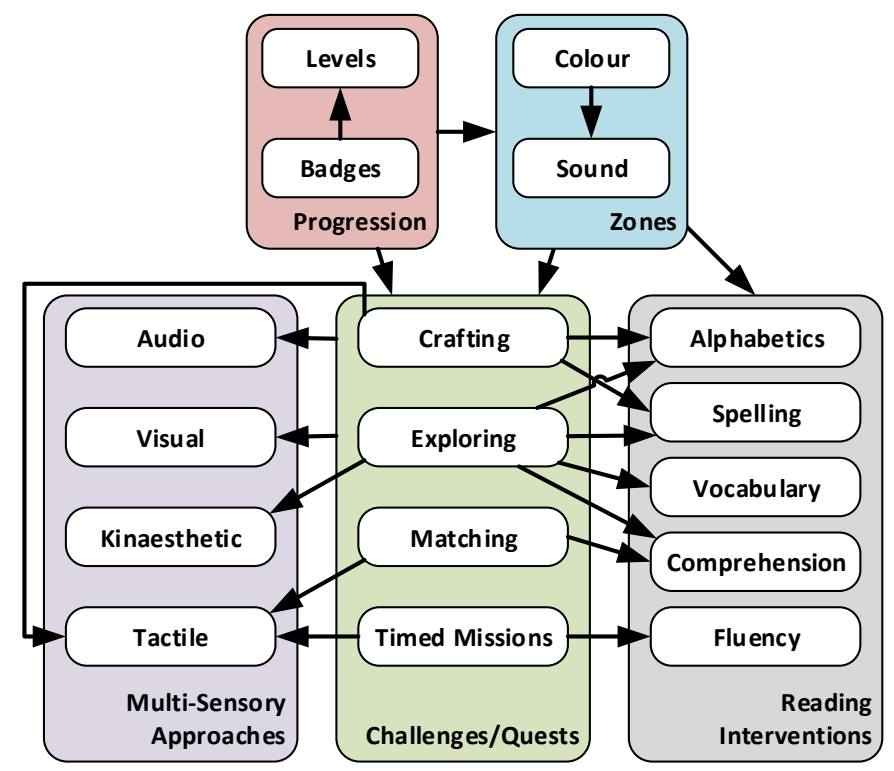

Fig. 1 A framework for gamified 3D environments

The game environment consists of 44 zones, which correspond to the 44 standard English phonemes [40]. Each zone is categorised and identified using colour and sound. Entering a zone will trigger audio of the zone's associated phoneme. The zones are populated with 3 dimensional graphemes of the associated phoneme. Each zone contains letters that make the same phoneme addressing audio sensory modality, as well as visual alphabetic component. This system aids the reader's categorisation of sound to grapheme correspondence, addressing the alphabetic principle. As an example, the $/ \mathrm{k} /$ zone would include "c" as in cat, " $\mathrm{k}$ " as in kilt, "ch" as in chrome and "cc" as in accent. The /a/ zone would include "a" as in cat, "ai" as in plaid and "au" as in laugh. The objective of this zonal system is to aid the learner's 
categorization of the relationship between grapheme and phoneme.

As the inclusion of all $1000+$ possible grapheme to phoneme constructs at start can overwhelm struggling learners, initially all zones will not be accessible to the player. Zones and more challenging phoneme to grapheme relationships become available through progression, adopting a system similar to the Hickey Multisensory Language Course [41]. High frequency irregular words would be introduced once a level of mastery has been reached. As the player's skills develop more zones will be unlocked within the environment. Levels of difficulty would be introduced through challenges with less time to complete, sentences to construct and the introduction of irregular phoneme to grapheme word constructs. Requiring the player to construct sentences would address the reading intervention area of fluency. A badge system is employed to address various components of reading intervention. Player could be awarded a vocabulary badge for completing a "noun" challenge by construction a set number of nouns, or rhyming badge for collecting a set number of words that rhyme.

Challenges or quests in the environment adopt exploration, crafting, matching and timed mission mechanics. Exploring the 3D environment and collecting resources utilises the kinaesthetic (movement) modality. Crafting the word heard or seen is the crafting quest, accessing audio or visual sensory modalities in isolation, or both simultaneously. Building a word within a set period of time is another challenge.

Player stores collected phoneme resources in an inventory. The inventory is categorized by phoneme where items in the inventory would utilise the same colour coding as the 3D environment. Interaction inventory items would trigger audio phonemes, aiding the categorization of underlying alphabetic principles [42]. The player crafts targets using collected grapheme resources. Tactile interaction with graphemes when placed in proximity to each other triggers blending of phonemes, supporting reading fluency. Once the word has been crafted it will be added to the learner's system vocabulary. Words within the vocabulary are colour coded according to word type (noun, verb, adjective, adverb, pronoun, preposition, conjunction). Player can toggle between phoneme based and word based colour coding, utilising audio, visual and tactile modalities. New quests/challenges are unlocked once a specified number of words have been added to the vocabulary, enabling progression to new zones.

\section{DEVElopment And Testing}

In order to implement the framework, it is important to involve the main stakeholders and users in the design and development of the system. As such, a focus group of volunteers from the BDA will be recruited (pending ethics approval). The focus group will be involved in the review of the proposed framework. The agreed design will then be implemented using a User-Centred Design approach [43] where the focus group will be involved in each iteration of the development cycle. All facilities and mechanics implemented will be passed on to the focus group to review. Their feedback is recorded and implemented back as requirements into the next cycle.

The final product will be tested through the contacts of the focus group and the BDA. Teachers who work with dyslexic students (preference with those already part of the focus group) are recruited on a voluntarily basis. The teachers will be trained in using the system and will use the system to work with their students. The focus of the test is on Key Stage 2 (7-10 years old) students, as often students are not assessed before this level. As the system is focused on aiding to read, the target students would also be at the moderate level of dyslexia. These students would be at Key Stage 2 but have fallen behind due to their learning difficulty and work at a Key Stage 1 level.

Whilst the system would be gathering data on its usage, such as interactions and clicks inter alia, the teachers will also be regularly interviewed using a semi-structured approach. The data from the system usage along with the qualitative data from the interviews will be thematically analysed in order to evaluate the effectiveness, usability and accessibility of the proposed framework.

\section{CONCLUSION AND FUTURE WORKS}

This paper proposes a framework for a gamified multisensory $3 \mathrm{D}$ environment to aid in reading with dyslexia. The framework is based on the finding in the literature and aims to use the abilities of gaming technology and 3D environments to increase engagement in students using the phonics method coupled with a computer assisted multisensory approach. The framework seeks to increase engagement, accessibility and availability of intervention whilst building on the strengths of the phonics approach.

The proposed framework has been presented and the methodology for implementation and testing of the framework through focus groups and user-centred design has been discussed. The research is currently awaiting ethical approval in order to form the focus group and then progress to the development stage.

\section{REFERENCES}

[1] R. L. Peterson and B. F. Pennington, "Developmental dyslexia," Lancet, vol. 379, no. 9830, pp. 1997-2007, 2012.

[2] BDA, "About British Dyslexia Association - Background.”.

[3] A. G. . "La Catts, H. W., Kamhi, The Connections Between Language and Reading Disabilities. Psychology Press, 2005.

[4] F. Ramus, "Neuroimaging sheds new light on the phonological deficit in dyslexia," Trends Cogn. Sci., vol. 18, no. 6, pp. 274-275, 2014.

[5] L. A. Barquero, N. Davis, and L. E. Cutting, "Neuroimaging of reading intervention: A systematic review and activation likelihood estimate meta-analysis," vol. 9, no. 1. 2014.

[6] J. W. Gilger, K. Allen, and A. Castillo, "Reading disability and enhanced dynamic spatial reasoning: A review of the literature," Brain Cogn., vol. 105, pp. 55-65, 2016.

[7] J. M. Maisog, E. R. Einbinder, D. L. Flowers, P. E. Turkeltaub, and G. F. Eden, "A meta-analysis of functional neuroimaging studies of dyslexia," vol. 1145. pp. 237-259, 2008. 
[8] J. S. Chall, "Learning to Read: The Great Debate 20 Years Later--A Response to \&quot;Debunking the Great Phonics Myth.\&quot;.," Phi Delta Kappan, vol. 70, no. 7, pp. 521-38, 1989.

[9] U. Goswami, "Sensory theories of developmental dyslexia: three challenges for research," Nat Rev Neurosci, vol. 16, no. 1, 2015.

[10] H. Scarborough and S. Brady, "Toward a Common Terminology for Talking About Speech and Reading: A Glossary of the \&quot;Phon\&quot; Words and Some Related Terms," vol. 34, no. 3 SAGE PublicationsSage CA: Los Angeles, CA, pp. 299-336, 2002.

[11] D. McGuinness, Early Reading Instruction: What Science Really Tells Us about How to Teach Reading. Cambridge, USA: MIT Press, 2006.

[12] K. S. Goodman, What's whole in whole language? Heinemann, 1986.

[13] N. I. of C. H. and H. D. (US) National Reading Panel (US), "Teaching children to read: An evidence-based assessment of the scientific research literature on reading and its implications for reading instruction."

[14] K. Galuschka, E. Ise, K. Krick, and G. Schulte-Körne, "Effectiveness of treatment approaches for children and adolescents with reading disabilities: A meta-analysis of randomized controlled trials," PLoS One, vol. 9, no. 2, 2014.

[15] S. S. Abdul Hamid, N. Admodisastro, and A. Kamaruddin, "A study of computer-based learning model for students with dyslexia," in 2015 9th Malaysian Software Engineering Conference, MySEC 2015, 2016.

[16] F. R. Vellutino, J. M. Fletcher, M. J. Snowling, and D. M. Scanlon, "Specific reading disability (dyslexia): what have we learned in the past four decades?," J. Child Psychol. Psychiatry, vol. 45, no. 1, pp. 2-40, Jan. 2004.

[17] M. J. Snowling, "From language to reading and dyslexia," Dyslexia, vol. 7, no. 1, pp. 37-46, Jan. 2001.

[18] G. McArthur et al., "Sight word and phonics training in children with dyslexia," J. Learn. Disabil., vol. 48, no. 4, pp. 391-407, 2015.

[19] S. Vaughn and G. Roberts, "Secondary interventions in reading; Providing additional instruction for students at risk," Teach. Except. Child., vol. 39, no. 5, pp. 40-46, 2007.

[20] J. Rose, "Identifying and teaching children and young people with dyslexia and literacy difficulties: an independent report," London, 2009.

[21] G. Reid, Dyslexia : a practitioners handbook. .

[22] G. M. Fernald, Remedial Techniques in Basic School Subjects. New York: McGraw-Hill, 1943.

[23] J. R. Birsh, Multisensory Teaching of Basic Language Skills, 3rd ed. Baltimore: Brookes Publishing Co, 2011.

[24] L. Moats, M. Farrell, and J. Birsh, "Multisensory structured language education," Multisensory Teach., 2005.

[25] R. Kamala, "Multisensory approach to reading skills of dyslexic students," IOSR J. Humanit. Soc. Sci. Ver. II, vol. 19, no. 5, pp. 32-34, 2014.

[26] S. Z. Ahmad, N. N. A. A. N. Ludin, H. M. Ekhsan, A. F. Rosmani, and M. H. Ismail, "Bijak Membaca - Applying Phonic Reading Technique and Multisensory Approach with interactive multimedia for dyslexia children," in CHUSER 2012 - 2012 IEEE Colloquium on Humanities, Science and Engineering Research, 2012.
[27] G. R. Lyon, S. E. Shaywitz, and B. A. Shaywitz, "A definition of dyslexia," Ann. Dyslexia, vol. 53, no. 1, pp. 1-14, Jan. 2003.

[28] T. E. Agency, “TEA Dyslexia Handbook,” Houston, 2014

[29] B. G. Jonassen, David H, Peck, Kyle L., Wilson, Learning with Technology: A Constructivist Perspective. New York: Merrill Publishing, 1999.

[30] Y. Cheng, H.-C. Chiang, J. Ye, and L. Cheng, "Enhancing empathy instruction using a collaborative virtual learning environment for children with autistic spectrum conditions," Comput. Educ., vol. 55, no. 4, pp. 1449-1458, 2010.

[31] B. M. Brooks, F. D. Rose, E. A. Attree, and A. Elliot-Square, "An evaluation of the efficacy of training people with learning disabilities in a virtual environment.," Disabil. Rehabil., vol. 24, no. 11-12, pp. 622$6,2002$.

[32] B. Garrett, T. Taverner, W. Masinde, D. Gromala, C. Shaw, and M. Negraeff, "A Rapid Evidence Assessment of Immersive Virtual Reality as an Adjunct Therapy in Acute Pain Management in Clinical Practice," Clin. J. Pain, vol. 30, no. 12, pp. 1089-1098, Dec. 2014.

[33] V. Enea, I. Dafinoiu, D. Opriş, and D. David, "Effects of Hypnotic Analgesia and Virtual Reality on the Reduction of Experimental Pain Among High and Low Hypnotizables," Int. J. Clin. Exp. Hypn., vol. 62, no. 3 , pp. 360-377, 2014.

[34] Tsang, M, Man, D, “A virtual reality-based vocational training system (VRVTS) for people with schizophrenia in vocational rehabilitation," Schizophr. Res., vol. 144, no. 1-3, pp. 51-62, Mar. 2013.

[35] M. R. Kandalaft, N. Didehbani, D. C. Krawczyk, T. T. Allen, and S. B. Chapman, "Virtual Reality Social Cognition Training for Young Adults with High-Functioning Autism," J. Autism Dev. Disord., vol. 43, no. 1, pp. 34-44, Jan. 2013

[36] Arora, Asit, Swords, Chloe, Khemani, Sam, Awad, Zaid, Darzid, Ara, Singh, Arvind Tolley,Neil, "Virtual reality case-specific rehearsal in temporal bone surgery: A preliminary evaluation," Int. J. Surg., vol. 12, no. 2, pp. 141-145, Feb. 2014.

[37] M. F. Levin, P. L. Weiss, and E. A. Keshner, "Emergence of Virtual Reality as a Tool for Upper Limb Rehabilitation: Incorporation of Motor Control and Motor Learning Principles," Phys. Ther., vol. 95, no. 3, pp. 415-425, Mar. 2015.

[38] G. H. Merchant, "3D virtual worlds as environments for literacy learning,” Educ. Res., vol. 52, no. 2, pp. 135-150, 2010.

[39] K. Kalyvioti and T. Mikropoulos, "Virtual environments and dyslexia: A literature review," Procedia Comput. Sci., 2014.

[40] M. K. Henry, Unlocking Literacy: Effective Decoding and Spelling Instruction. Baltimore USA: Brookes Publishing Co, 2003.

[41] K. Hickey and M. Combley, The Hickey multisensory language course. Whurr, 2001

[42] S. Carreker, Multisensory grammar and written composition. Houston, Texas: Neuhaus Education Center, 1993.

[43] R. J. Pagulayan, K. Keeker, D. Wixon, R. L. Romero, and T. Fuller, "The Human-computer Interaction Handbook," J. A. Jacko and A. Sears, Eds. Hillsdale, NJ, USA: L. Erlbaum Associates Inc., 2003, pp. 883-906. 